\title{
THE STONE-WEIERSTRASS THEOREM
}

\section{LOUIS DE BRANGES}

Let $S$ be a locally compact Hausdorff space and let $C(S)$ be the continuous complex valued functions which (in the noncompact case) vanish at infinity. Let $E$ be a vector subspace of $C(S)$ which is closed under complex conjugation. We ask for conditions that $E$ be uniformly dense in $C(S)$. (The hypothesis that $E$ be closed under complex conjugation allows us to reduce the problem to one for real valued functions. The reader may prefer to recast the discussion in that context.)

We have chosen to present our result as a lemma towards a proof of the Stone-Weierstrass theorem. This approach not only sheds an interesting light on that theorem, but will help the reader understand the nature of the lemma. However, the discussion of Stone [3] remains the most direct and, when all details are considered, the shortest proof of the Stone-Weierstrass approximation theorem. A more serious application of the lemma will be made later in a paper on the Bernstein approximation problem.

Let $U(E)$ be the set of all real valued measures $\mu$ on the Borel subsets of $S$, with total variation at most 1 , such that for every $f$ in $E$, $\int f d \mu=0$. Consider $U(E)$ in the weak topology induced by $C(S)$ under integration. Then $U(E)$ is a compact, convex set, and if $E$ is not dense in $C(S), U(E)$ contains a nonzero element (Loomis [2, pp. 22-23, pp. 29-47]). By the Krein-Milman theorem, $U(E)$ is the weakly closed convex span of its extreme points (Krein-Milman [1]).

Lemma. Let $\mu$ be a nonzero extreme point of $U(E)$. If $g$ is a Borel measurable complex valued function on $S$ which is essentially bounded with respect to $\mu$, such that for every $f$ in $E, \int f g d \mu=0$, then $g$ is equal a.e. with respect to $\mu$ to a constant.

The conclusion of the lemma has this interesting interpretation. For each complex valued Borel measurable function $k$ on $S$ such that $\int|k d \mu|<\infty$ and $\int k d \mu=0$, there is a sequence $\left(f_{n}\right)$ in $E$ such that $\lim _{n} \int\left|k-f_{n}\right||d \mu|=0$. We are using the fact that if $E$ is a vector subspace of a Banach space $X$, the norm closure of $E$ is equal to the orthogonal complement $\left(E^{\perp}\right)^{\perp}$ in $X$ of the orthogonal complement $E^{\perp}$ in $X^{*}$ of $E$. Here $X^{*}$ is the adjoint Banach space to $X$. This matter

Received by the editors September 22, 1958 and, in revised form, February 11, 1959. 
is related to the Hahn-Banach theorem and is discussed in the same Loomis reference.

Proof of Lemma. It is obvious that if $\mu$ is a nonzero extreme point of $U(E), \int|d \mu|=1$. We will prove the lemma by showing that if $\mu$ is an element of $U(E)$ such that $\int|d \mu|=1$ and if there exists a Borel measurable function $g$, which is essentially bounded with respect to $\mu$ and is not equal a.e. to a constant with respect to $\mu$, such that for every $f$ in $E, \int f g d \mu=0$, then $\mu$ is not an extreme point of $U(E)$. Note that $\mu$ is a real valued measure and that $E$ is assumed closed under complex conjugation. Therefore, if such a function $g$ exists, either $\operatorname{Re} g$ or Im $g$ is a real valued function meeting the same specifications. We can suppose then that $g$ is real valued. By adding a positive constant to $g$, we can suppose that $g$ is positive valued a.e. with respect to $\mu$. By multiplying by a positive constant, we can suppose that $\int g|d \mu|=1$. Let $\lambda^{-1}$ be an essential bound for $g$ with respect to $\mu$. Since $g$ is essentially bounded, $\lambda>0$. If it were true that $\lambda \geqq 1$, then we would have

$$
\int|1-g||d \mu|=\int|d \mu|-\int g|d \mu|=1-1=0,
$$

which contradicts the hypothesis that $g$ is not equivalent to a constant a.e. with respect to $\mu$. Therefore, $0<\lambda<1$. Let $\mu_{1}$ and $\mu_{2}$ be the measures defined on Borel sets $A$ of $S$ by

$$
\begin{aligned}
& \mu_{1}(A)=\int_{A} g d \mu, \\
& \mu_{2}(A)=\int_{A} \frac{1-\lambda g}{1-\lambda} d \mu .
\end{aligned}
$$

Then it is obvious that $\mu_{1}$ and $\mu_{2}$ are in $U(E)$ and are not equal to $\mu$ and that $\mu=\lambda \mu_{1}+(1-\lambda) \mu_{2}$. Therefore, $\mu$ is not an extreme point of $E$. Q.E.D.

Stone-Weierstrass Theorem. If (1) for each point $P$ of $S$ there is some element $f$ of $E$ such that $f(P) \neq 0$,

(2) for each pair $P, Q$ of distinct points of $S$ there is an element $f$ of $E$ such that $f(P) \neq f(Q)$,

(3) whenever $f, g$ are in $E$ the product $f g$ is in $E$, then $E$ is uniformly dense in $C(S)$.

Proof of Theorem. Let $E$ be a vector subspace of $C(S)$ which is closed under complex conjugation and satisfies (2) and (3) and yet is not dense in $C(S)$. We will prove the theorem by showing that (1) is not satisfied. In fact, $U(E)$ then has a nonzero element and hence a 
nonzero extreme point $\mu$. Let $g$ be any element of $E$. By (3) for every $f$ in $E, f g$ is in $E$, and hence $\int f g d \mu=0$. By the lemma, $g$ is equal a.e. with respect to $\mu$ to a constant. But by the continuity of $g, g$ is equal to a constant on the support of $\mu$. Since for a nonzero extreme point $\int|d \mu|=1$, we have the formula $g(P)=\int g|d \mu|$ for every $g$ in $E$ and every point $P$ in the support of $\mu$. But now (2) implies that $\mu$ is supported at a single point $P$. Since for every $g$ in $E, \int g d \mu=0$ and $|\mu(\{P\})|=1 \neq 0$, we have that for every $g$ in $E, g(P)=0$. This contradicts (1). Q.E.D.

\section{REFERENCES}

1. M. Krein and D. Milman, On extreme points of regular convex sets, Studia Math. vol. 19 (1940) pp. 133-138.

2. L. H. Loomis, Introduction to abstract harmonic analysis, New York, van Nostrand, 1953.

3. M. H. Stone, The generalized Weierstrass approximation theorem, Math. Mag. vol. 21 (1948) pp. 167-184, 237-254.

Lafayette College 